\title{
Implemented a wireless communication system for VGA capsule endoscope
}

\author{
Yeon-Kwan Moon ${ }^{\mathrm{a}}$, Jyung Hyun Lee ${ }^{\mathrm{b}}$, Hee-Joon Park ${ }^{\mathrm{c}}$, Jin-Ho Cho $^{\mathrm{d}}$ and Hyun-Chul Choi ${ }^{\mathrm{d}, *}$ \\ ${ }^{a}$ Samsung Electronics, 94-1, Imsoo-dong, Gumi-si, Gyeongsanbuk-do, Korea \\ ${ }^{b}$ Department of Biomedical Engineering, Kyungpook National University Hospital, 807 Hogukno, \\ Buk-gu, Daegu, Korea \\ ${ }^{c}$ Department of Biomedical Engineering, School of Medicine, Keimyung University, 1095 Dalgubeol- \\ daero, Daegu, Korea \\ ${ }^{d}$ School of Electronics Engineering, College of IT Engineering, Kyungpook National University, 80 \\ Daehakro, Bukgu, Daegu, Korea
}

\begin{abstract}
Recently, several medical devices that use wireless communication are under development. In this paper, the small size frequency shift keying (FSK) transmitter and a monofilar antenna for the capsule endoscope, enabling the medical device to transmit VGA-size images of the intestine. To verify the functionality of the proposed wireless communication system, computer simulations and animal experiments were performed with the implemented capsule endoscope that includes the proposed wireless communication system. Several fundamental experiments are carried out using the implemented transmitter and antenna, and animal in-vivo experiments were performed to verify VGA image transmission.
\end{abstract}

Keywords: Capsule endoscope, wireless communication, antenna

\section{Introduction}

Recently, several types of wireless medical devices are under development. The key requirements of these devices are small size and low power consumption [1]. In addition, high-speed data transmission and low attenuation are required in wireless capsule endoscopy applications.

Various capsule endoscopes have been developed after the success of the M2A capsule (Given imaging, Israel) [2,3]. Capsule endoscopy does not necessitate the administration of sedatives because it is a safe procedure that causes no pain. Unlike, conventional wired endoscopes, capsule endoscope allow the viewing of the entire small intestine [4]. Commercial capsule endoscopes include PillCam SB (Given imaging, Israel), EndoCapsule (Olympus, Japan), and MiroCam (IntroMedic, Korea). The communication method employed in a capsule endoscope is RF communication or human body communication. Because of the uncertainty regarding the safety of the human body communication method, the RF communication method is widely used. The image size that can be achieved with a com-

\footnotetext{
* Corresponding author: Hyun-Chul Choi, School of Electronics Engineering, College of IT Engineering, Kyungpook National University, 80 Daehakro, Bukgu, Daegu, Korea. Tel.: +82-53-427-5538; Fax: +82-53-427-5539; E-mail: hcchoi@ee.knu.ac.kr.
} 
mercial capsule endoscope is limited to less than 102,400 pixels. The reasons for this limitation of the image size are the limited data transmission speed and the attenuation of RF signals in the human body.

In this paper, a small-size frequency shift keying (FSK) transmitter and a monofilar antenna that can transmit VGA-size images of the intestine are designed and implemented for the capsule endoscope. To verify the functionality of the proposed wireless communication system, computer simulations and animal experiments were performed using the implemented capsule endoscope. The computer simulation software HFSS was used and the capsule endoscope that included the proposed transmitter and antenna was implemented for animal experiments.

\section{Method}

In the capsule endoscope, uncompressed data of a single VGA image $(640 \times 480$ pixels $)$ is 14.4 Mbits per frame. Therefore, the target data transmission rate was set as $20 \mathrm{Mbps}$ in the proposed communication system for the capsule endoscope. To achieve $20 \mathrm{Mbps}$ data transmission, careful selection of the communication frequency, transmitting power, system size, frequency interference, and modulation method are required.

\subsection{Selection of RF communication specifications}

Communication channels that pass through the human body are heterogeneous, anisotropic, and lossy [5]. The permittivity and the conductivity of human organs and tissues change depending on the frequency, unlike in a normal dielectric structure.

The permittivity is high in the low-frequency band, but the conductivity is high in the highfrequency band. This means that the attenuation is high in the high-frequency region and significant signal distortion can occur in low-frequency region. The intrinsic impedance of the human body is calculated as follows;

$$
\eta=|\eta| \angle \theta=\sqrt{\frac{j \omega \mu}{\sigma+j \omega \varepsilon}}=\sqrt{\frac{\omega^{2} \mu \varepsilon+j \omega \mu \sigma}{\sigma^{2}+(\omega \varepsilon)^{2}}}
$$

where the dielectric constant is $8.85 \times 10^{-12} \mathrm{~F} / \mathrm{m}$ and the permeability constant is $4 \pi \times 10^{-7} \mathrm{H} / \mathrm{m}$. The intrinsic impedance of the human organ is roughly $100 \Omega$. A comparison of relevant characteristics for different transmission frequencies is provided in Table 1.

Table 1

Comparison of characteristics with transmission frequency

\begin{tabular}{lllll}
\hline Frequency $[\mathrm{MHz}]$ & 400 & 900 & 1200 & 2400 \\
\hline MPE $[\mathrm{dBm}]$ & 1.25 & 4.78 & 6.02 & 6.99 \\
Attenuation & Better & Good & Good & Worse \\
Power & Better & Good & Good & Worse \\
Antenna size & Bad & Good & Good & Better \\
Interference & Alarm Systems & Cellular Networks & - & W-LANs \\
\hline
\end{tabular}


From Table 1, it is seen that the communication frequency appropriate for the capsule endoscope is $1.2 \mathrm{GHz}$, considering the maximum permissible exposure (MPE), attenuation in the human body, antenna size and interference from other systems or networks. The value of MPE is limited to $4 \mathrm{~mW} / \mathrm{cm}^{2}$ at $1.2 \mathrm{GHz}$ by ICNIRP [6]. If the antenna radius is $0.5 \mathrm{~cm}$ and the surface area is $3.14 \mathrm{~cm}^{2}$ then the maximum transmission power is $12.56 \mathrm{~mW}$. The modulation method is selected the FSK for small size transmitter, because its circuit is simple and constant envelope characteristic is good.

The loss of RF signals occurs in the human body due to the attenuation in body tissues and the reflection at the boundary of tissues. The attenuation is determined from the angular frequency $\omega$, the permittivity $\varepsilon$, the conductivity $\sigma$, and the permeability $\mu$ as follows $[7,8]$;

$$
\alpha=-0.0868 \times \omega \sqrt{\frac{\mu \varepsilon}{2}\left\{1+\left(\frac{\sigma}{\omega \varepsilon}\right)^{2}-1\right\}}[\mathrm{dB} / \mathrm{cm}]
$$

Computer simulation is performed using HFSS (Ansoft, USA), and Eq. (2) is used for calculating attenuation and reflection. For simulation and calculation, 4 target sections and several paths were used as Figure 1.

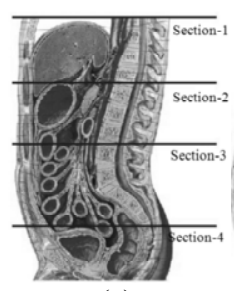

(a)

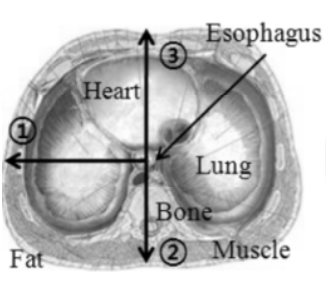

(b)

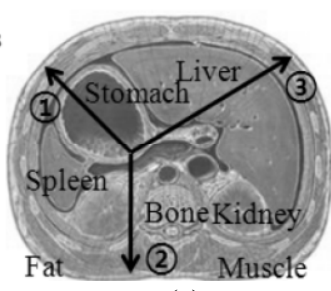

(c)

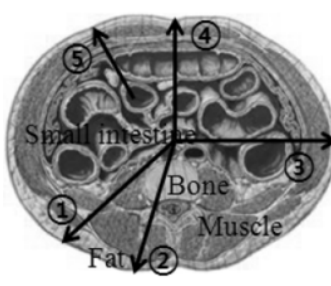

(d)

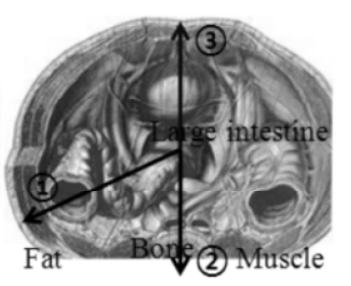

(e)

Fig. 1. Cross-sectional view with transmission paths: (a) The side view, and (b) the 1st, (c) the 2nd, (d) the 3rd, and (e) the 4th sections.

Table 2

Attenuation of RF signals in the human body at $1.2 \mathrm{GHz}$

\begin{tabular}{|c|c|c|c|c|}
\hline Section and Path & $\begin{array}{l}\text { Reflection } \\
\text { loss [dB] }\end{array}$ & $\begin{array}{l}\text { Body attenuation loss } \\
\text { [dB] }\end{array}$ & Calculated total loss $[\mathrm{dB}]$ & $\begin{array}{l}\text { Simulated total loss } \\
{[\mathrm{dB}]}\end{array}$ \\
\hline Section 1, Path 1 & 1.7193 & 29.6027 & 31.322 & 30.2381 \\
\hline Section 1, Path 2 & 2.5458 & 17.2753 & 19.8211 & 15.6787 \\
\hline Section 1, Path 3 & 1.1788 & 41.2826 & 42.4614 & 39.8083 \\
\hline Section 2, Path 1 & 1.1807 & 35.1517 & 36.3324 & 33.5085 \\
\hline Section 2, Path 2 & 1.1828 & 39.4023 & 40.5851 & 38.5289 \\
\hline Section 2, Path 3 & 1.2145 & 51.1461 & 52.3606 & 51.0286 \\
\hline Section 3, Path 1 & 1.1988 & 41.1894 & 42.3882 & 40.6385 \\
\hline Section 3, Path 2 & 2.5795 & 37.9624 & 40.5419 & 34.4485 \\
\hline Section 3, Path 3 & 1.1988 & 76.3264 & 77.5252 & 75.6887 \\
\hline Section 3, Path 4 & 1.1988 & 52.645 & 53.8438 & 53.1381 \\
\hline Section 3, Path 5 & 1.1988 & 34.8431 & 36.0419 & 41.7200 \\
\hline Section 4, Path 1 & 1.1746 & 48.1454 & 49.3200 & 51.9083 \\
\hline Section 4, Path 2 & 2.4447 & 26.6563 & 29.1010 & 30.4188 \\
\hline Section 4, Path 3 & 1.1746 & 34.7906 & 35.9652 & 36.3585 \\
\hline
\end{tabular}


The location of the capsule endoscope is the esophagus in section 1, the stomach in section 2, the small intestine in section 3, and the large intestine in section 4. Simulation results are tabulated in Table 2

The maximum attenuation was $77.5252 \mathrm{~dB}$ in Table 2. However, the attenuations in the other paths for the section were less than $54 \mathrm{~dB}$. So, the total loss was $64 \mathrm{~dB}$ which included human body loss $(-54$ $\mathrm{dB})$ and antenna gain $(-10 \mathrm{~dB})$. The required minimum sensitivity of the proposed receiver was -73 $\mathrm{dBm}$, because the maximum transmitting power limited to $10.98 \mathrm{dBm}$, the total loss was $64 \mathrm{~dB}$ in human body and the system margin was $20 \mathrm{~dB}$ for the minimum sensitivity.

The simplicity of modulation and demodulation schemes, robustness at varying noise levels, and small size should be considered for the selection of the modulation method. The FSK modulation scheme is selected for the proposed system as the scheme preserves constant envelope characteristics and requires a simple signal processing circuit.

\subsection{Design of transmitter and antenna}

The proposed transmitter is based on the Clapp oscillator and a buffer. Generally, degradation in phase noise characteristics is expected because of the low Q-factor in the small-size design of the Clapp oscillator. To solve the low Q-factor problem, the proposed transmitter is designed with an inductor implemented by a micro-strip line, a varactor with a high Q-factor, and a transistor with a high transition frequency and good noise characteristic. The designed voltage controlled oscillator (VCO) circuit is shown in Figure 2(a). The load impedance variation is common cause of phase noise degradation and frequency stability degradation in the VCO. So, a buffer amplifier is added between the VCO and the antenna to solve this problem. The designed buffer amplifier circuit is shown in Figure 2(b).

The resonator of the designed VCO circuit consists of a high Q-factor varactor diode BBY53-03W (Infineon, USA) and a micro-strip line inductor of a small size and a high Q-factor, as shown in Figure 3. The calculated inductance and the Q-factor of the designed micro-strip line inductor are $1.344 \mathrm{nH}$ and 183.744 respectively. Computer simulation of the designed VCO was performed using ADS (advanced design system) (Agilent, USA). The output power is $-4.353 \mathrm{dBm}$ and the phase noise was $112.9 \mathrm{dBc} / \mathrm{Hz}$ at $1.204 \mathrm{GHz}$. The gain and the isolated gain of the designed buffer are calculated as 15 $\mathrm{dB}$ and $-23 \mathrm{~dB}$ by simulation using ADS.

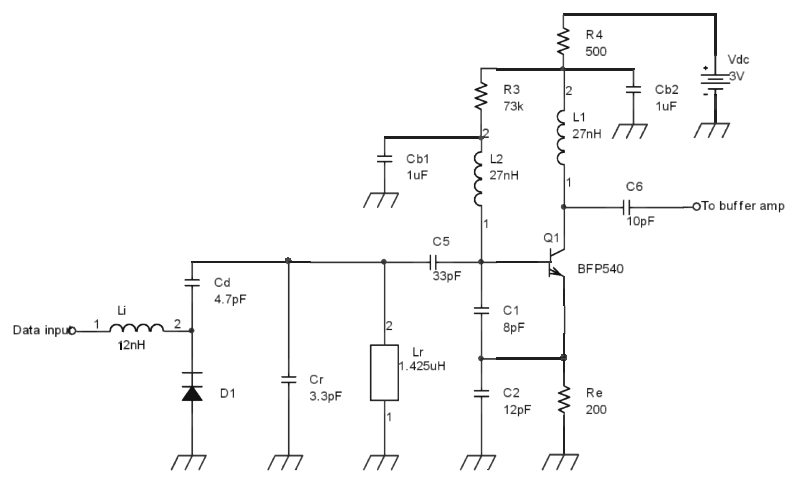

(a)

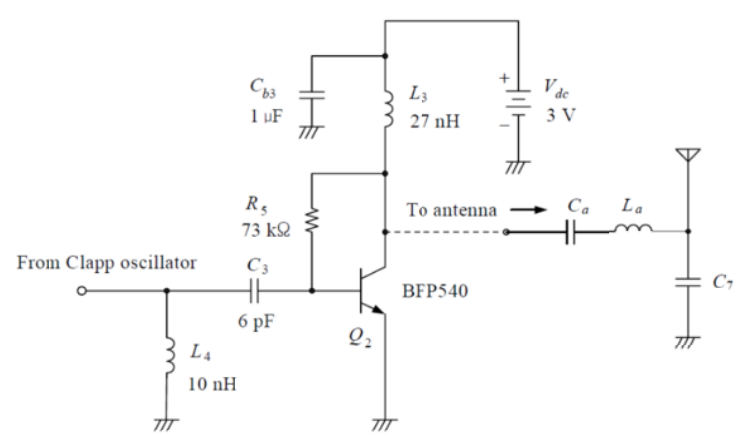

(b)

Fig. 2. Circuit diagram of (a) designed VCO circuit and (b) designed buffer amplifier circuit. 


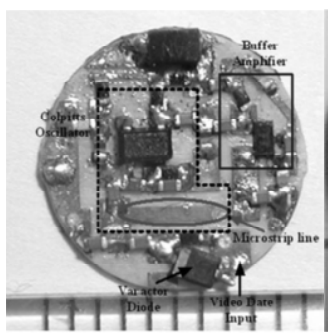

(a)

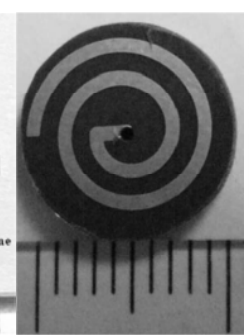

(b)

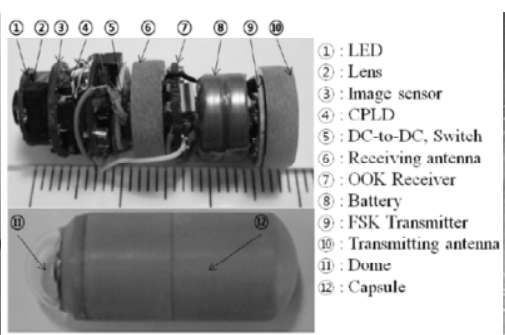

(c)

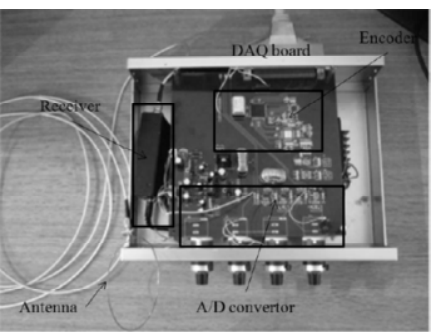

(d)

Fig. 3. Implemented (a) transmitter, (b) antenna, (c) capsule endoscope with proposed system, and (d) receiver.

Table 3

Specifications of the optimized small monofilar antenna

\begin{tabular}{ll}
\hline Specification & Value \\
\hline Frequency $[\mathrm{MHz}]$ & 1200 \\
Diameter [mm] & 10 \\
Line width $[\mathrm{mm}]$ & 0.7 \\
Initial radius [mm] & 1.5 \\
Rate of radius increase & 1.8 \\
Number of turn & 2 \\
\hline
\end{tabular}

The size of the transmitting antenna needs to be small and its communication bandwidth wide for the antenna to be used for a capsule endoscope. The monofilar antenna, a planar spiral antenna with a helical structure, is proposed to secure small size and wide bandwidth. Computer simulation that determines the antenna structure for achieving the optimum radiation efficiency is performed using HFSS (Ansoft, USA). The maximum radiation efficiency according to the line width, number of turns, spiral increment, initial radius, radius increase rate, and thickness of substrate is simulated with a dielectric constant 3.38 and a thickness of $3 \mathrm{~mm}$. The specifications of the optimized small monofilar antenna are listed in Table 3.

\section{Experiments and results}

\subsection{Implemented proposed system}

To perform the wireless transmission experiments, the proposed system that includes the designed transmitter and the antenna, shown in Figure 4, were implemented. The implemented capsule endoscope has a VGA grade CMOS image sensor (PO2030N, Pixelplus), a CPLD controller (XCR3064, Xilinx) and battery. The size of the implemented capsule is $12 \mathrm{~mm}$ (diameter) and $27 \mathrm{~mm}$ (length).

The diameter and the thickness of implemented transmitter are $10 \mathrm{~mm}$ and $1.6 \mathrm{~mm}$ respectively. The specifications of the implemented transmitter shown in Figure 3 are listed in Table 4.

The output power of the implemented transmitter was $0.5 \mathrm{dBm}$. The phase noise of the transmitter measured at $100 \mathrm{kHz}$ offset is $-106 \mathrm{~dB} / \mathrm{Hz}$, and its harmonic suppression is $-28.83 \mathrm{dBc}$, as shown in Figure 4 and listed in Table 4 . The modulated output spectrum and the received signal are shown in Figure 5, for the input signal rate of 20 Mbps. 


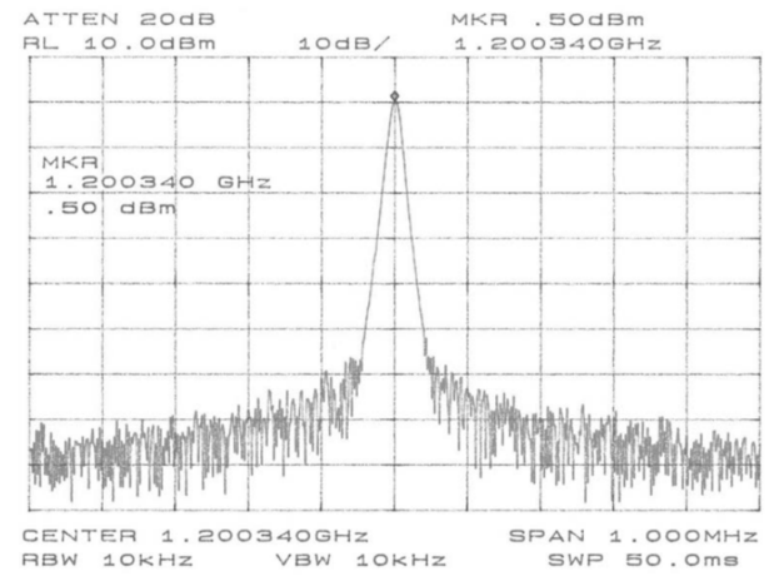

(a)

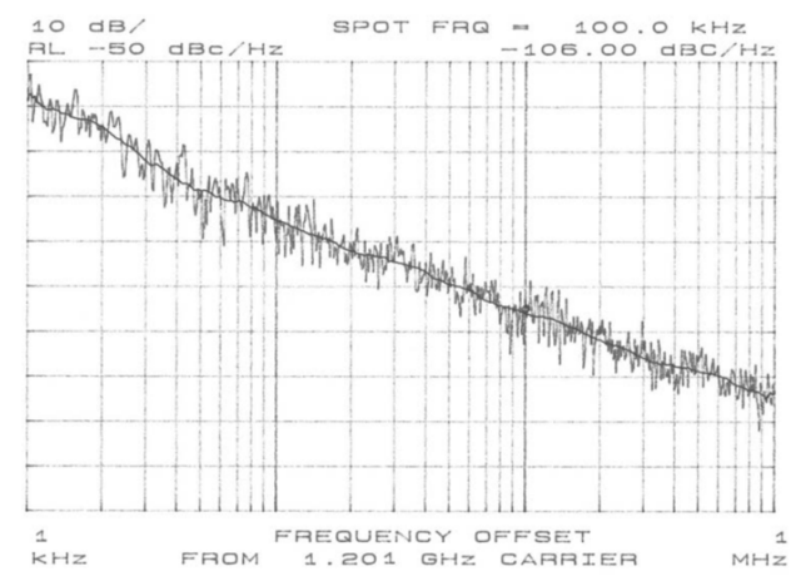

(b)

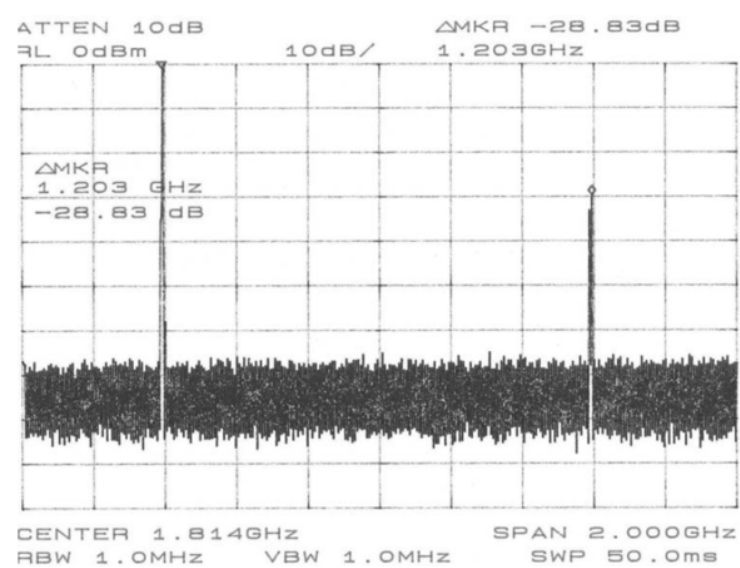

(c)

Fig. 4. Measurement results of the implemented transmitter: (a) output power, (b) phase noise, and (c) harmonic characteristics.

Table 4

Specifications of the implemented transmitter

\begin{tabular}{lll}
\hline Part & Specification & Measurement value \\
\hline \multirow{4}{*}{ VCO } & Frequency range [MHz] & $1202 \sim 1262$ \\
& Turning voltage [V] & $0 \sim 3$ \\
& Output Power [dBm] & -6 \\
\multirow{3}{*}{ Buffer amp. } & Phase noise (@100 KHz offset)[dBc/Hz] & -108.83 \\
& Gain [dB] & 12.684 \\
& Isolation [dB] & -27.757 \\
\multirow{2}{*}{ FSK Tx } & Bias [V]/[mA] & $3 / 5$ \\
& Modulation frequency [MHz] & $1240 \sim 1261$ \\
& Phase noise (@100 KHz offset)[dBc/Hz] & -106 \\
& $2^{\text {nd }}$ harmonic [dBc] & -28.83 \\
\hline
\end{tabular}




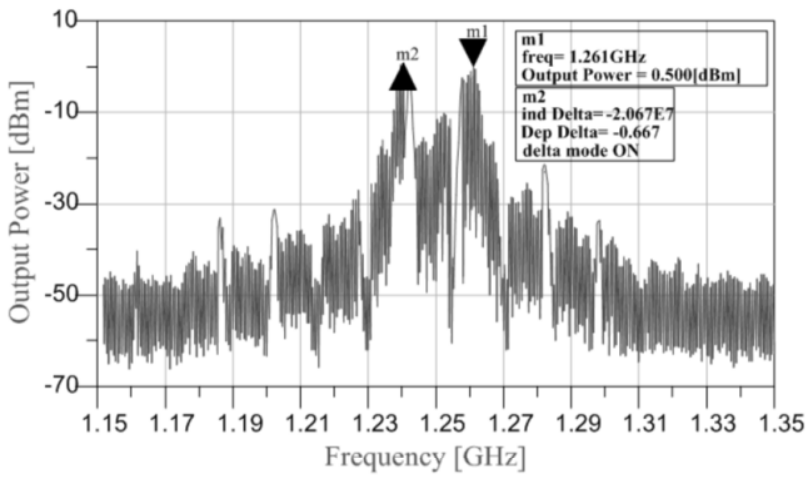

(a)

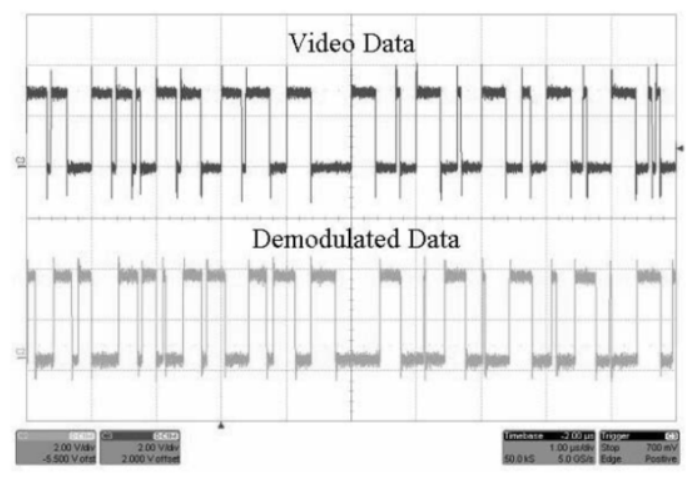

(b)

Fig. 5. The measurement results using the implemented transmitter; (a) modulated spectrum and (b) demodulated signal.

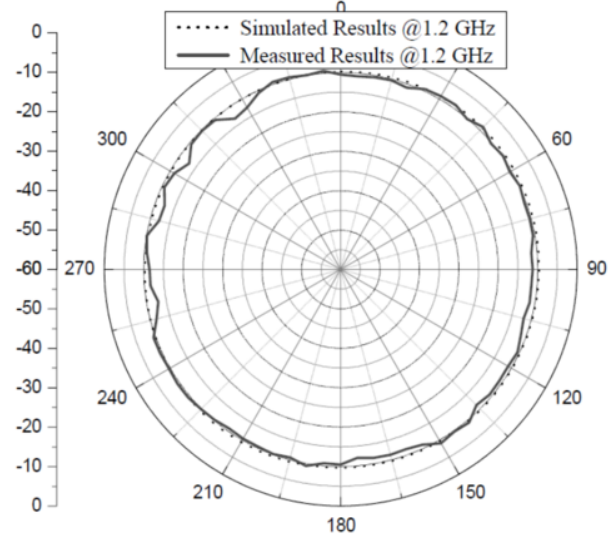

(a)

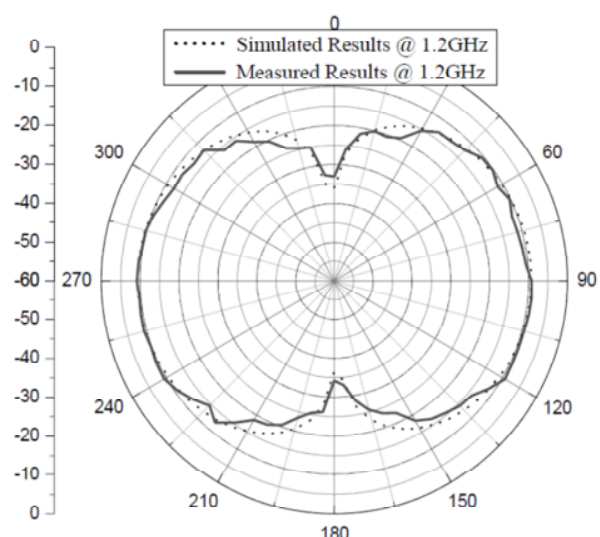

(b)

Fig. 6. Measured and simulated radiation patterns in (a) $\Phi$ direction and (b) $\theta$ direction.

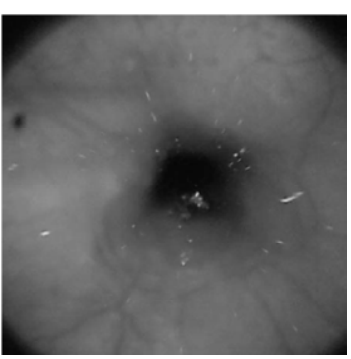

(a)

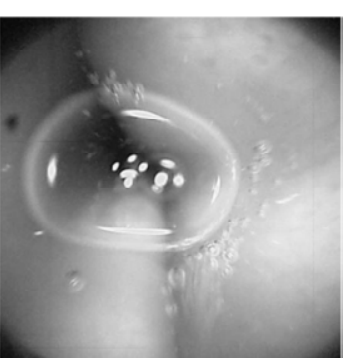

(b)

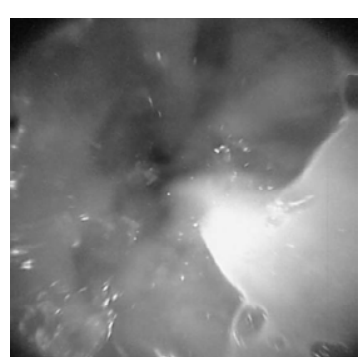

(c)

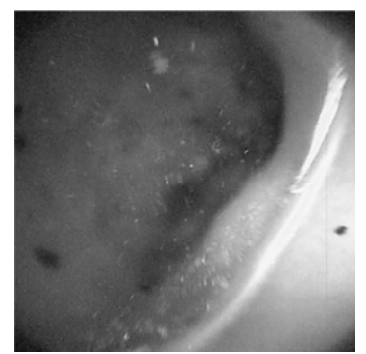

(d)

Fig. 7. Reconstructed images of (a) the esophagus, (b) the stomach, (c) the small intestine, and (d) the large intestine.

The frequency variation of the implemented transmitter is $20 \mathrm{MHz}$ and its power variation is $0.6 \mathrm{~dB}$, as shown in Figure 5(a). The received signal is restored same signal as the transmitted signal in Figure 5(b). 
The implemented planar small monofilar antenna is fabricated by an etching method of accuracy 0.1 $\mathrm{mm}$. The material of the antenna was RT/duroid 6002 laminate (Rogers, USA), and the thickness of the board is $3 \mathrm{~mm}$. The thickness of the conductor is $0.017 \mathrm{~mm}$, and the permittivity and the dielectric loss tangent are 2.94 and 0.0012 respectively. The radiation pattern and the gain of the implemented antenna are measured in the electromagnetic shielding chamber. The measured and simulated radiation patterns are shown in Figure 6.

The measured radiation pattern is almost the same as the simulated pattern. The measured gain of the implemented planar structure small monofilar antenna was $-8.92 \mathrm{~dB}$.

\subsection{In-vivo experiments and results}

The in-vivo experiments were performed to verify the proposed system in the animal laboratory at Yonsei University. The implemented capsule that includes the proposed system shown in Figure 3(c) was inserted into the esophagus of a swine, which was anesthetized. The restored images from the invivo experiment are shown as Figure 7.

\section{Discussion and conclusion}

In this paper, the RF communication system is proposed for VGA capsule endoscope. The MPE, attenuation of human body, permissible transmitting output power, power consumption, and antenna gain are considered during the design stage of the proposed system. The communication frequency is selected as $1.2 \mathrm{GHz}$ on the basis of RF signals in human body as well as the size of the antenna. The selected antenna type is the planar-structure small monofilar antenna according to the size of antenna and bandwidth of communication. The proposed transmitter and antenna are designed and verified through computer simulations. The output power of the implemented FSK transmitter is $0.5 \mathrm{dBm}$, and the measured antenna gain is $-8.92 \mathrm{~dB}$.

To verify the proposed system, several fundamental experiments were performed using the implemented transmitter and antenna. From the results of experiments, it was verified that the implemented system exhibits satisfactory efficiency and performance. Animal in-vivo experiments were performed to verify the VGA image transmission. The implemented VGA capsule endoscope with the proposed transmitter and antenna transmitted high-quality VGA images at the rate of 1.4 frames per second. These images are of better quality and bigger size than the images obtained with existing commercial capsule endoscopes such as PillCam and MiroCam.

The proposed techniques have sufficient merit for implementing the communication system in the medical device. If the circuit of the proposed transmitter can be fabricated on a single chip, then the size and the power consumption of the device can be further reduced. Once these present limitations are overcome, the capsule endoscope will be used more widely in the field of diagnosis.

\section{Acknowledgement}

This work was supported by the National Research Foundation of Korea (NRF) grant funded by the Korea government (No. NRF-2012R1A1A2006475). 


\section{References}

[1] H.Y. Liua, P.P Qiaoa, X.L. Wua, W. Lia, Y.L. Aoa, Z. Jiaa and X.T. Pi, A smart capsule system of gastric occult blood detection, Bio-Medical Materials and Engineering 24 (2014), 519-528.

[2] Y.K. Moon, J.H. Lee, H.J. Park, J. Lee, J.J. Ryu, S.H. Woo, M.-K. Kim, C.-H. Won, T. Kim, J.-H. Cho and H.C. Choi, Fabrication of the wireless systems for controlling movements of the electrical stimulus capsule in the small intestines, IEICE Transactions 90 (2007), 586-593.

[3] J.H. Lee, Y.K. Moon, Y.H. Yoon, H.J. Park, C.-H. Won, H.C. Choi and J.-H. Cho, CPLD based bi-directional wireless capsule endoscopes, IEICE Transactions 90 (2007), 694-697.

[4] J.H. Lee and J.H. Cho, Wireless transmission method for VGA capsule endoscopy using Manchester encoding, Biomed. Eng. Lett. 3 (2013), 250-257.

[5] Arthur T. Hubbard, Encyclopedia of Surface and Colloid Science, Marcel Dekker. Inc., NY, 2002, pp. $2643-2652$.

[6] International Commission on Non-Ionizing Radiation Protection, Guidelines for limiting exposure to time-varying electric, magnetic, and electromagnetic fields (up to $300 \mathrm{GHz}$ ), Health Physics 74 (1998), 494-522.

[7] C. Gabriel, S. Gabriel and E. Corthout, The dielectric properties of biological tissues: I. Literature survey, Physics in Medicine and Biology 41 (1996), 2231-2249.

[8] S. Gabriel, R. Lau and C. Gabriel, The dielectric properties of biological tissues: II. Measurements in the frequency range $10 \mathrm{~Hz}$ to $20 \mathrm{GHz}$, Physics in Medicine and Biology 41 (1996), 2251-2269. 